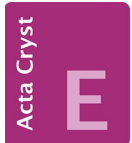
COMMUNICATIONS

ISSN 2056-9890

\section{Crystal structure of $\left[1,1^{\prime}\right.$-biphenyl]-2,2'- dicarbonitrile}

\author{
Gihaeng Kang, Tae Ho Kim,* Youngeun Jeon and Jineun \\ Kim*
}

Department of Chemistry and Research Institute of Natural Sciences, Gyeongsang National University, Jinju 660-701, Republic of Korea. ${ }^{*}$ Correspondence e-mail: thkim@gnu.ac.kr, jekim@gnu.ac.kr

Received 12 May 2015; accepted 19 May 2015

Edited by M. Weil, Vienna University of Technology, Austria

The complete molecule of the title compound, $\mathrm{C}_{14} \mathrm{H}_{8} \mathrm{~N}_{2}$, is generated by a twofold rotation axis located at the midpoint of the biphenyl $\mathrm{C}-\mathrm{C}$ bond. The dihedral angle between the symmetry-related phenyl rings is $46.16(3)^{\circ}$. In the crystal, molecules are linked by slipped parallel $\pi-\pi$ interactions [centroid-centroid distance $=3.9451$ (7) $\AA$, normal distance = 3.6293 (5) $\AA$, slippage $1.547 \AA$ ] , forming columns along the $b$ axis direction.

Keywords: crystal structure; biphenyl; $\pi-\pi$ contacts.

CCDC reference: 1401615

\section{Related literature}

The title compound has been used as a reactant for phthalocyanine synthesis (Shimizu et al., 2011, 2014). Related crystal structures were reported by Furukawa et al. (2008) and Paek et al. (1989). For synthetic details, see: Wu et al. (2007).

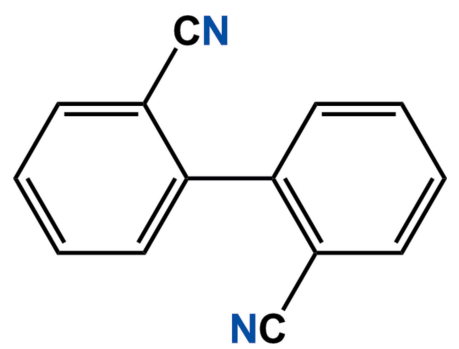

\section{Experimental}

2.1. Crystal data

$\mathrm{C}_{14} \mathrm{H}_{8} \mathrm{~N}_{2}$

$M_{r}=204.22$

Monoclinic, $C 2 / c$

$a=15.7839$ (9) §

$b=3.9451$ (2) $\AA$

$c=16.6079(9) \AA$

$\beta=101.630(3)^{\circ}$

$$
\begin{aligned}
& V=1012.93(9) \AA^{3} \\
& Z=4 \\
& \text { Mo } K \alpha \text { radiation } \\
& \mu=0.08 \mathrm{~mm}^{-1} \\
& T=173 \mathrm{~K} \\
& 0.43 \times 0.12 \times 0.06 \mathrm{~mm}
\end{aligned}
$$

\subsection{Data collection}

Bruker APEXII CCD diffractometer

Absorption correction: multi-scan (SADABS; Bruker, 2009)

$T_{\min }=0.966, T_{\max }=0.995$

4708 measured reflections 1157 independent reflections 988 reflections with $I>2 \sigma(I)$ $R_{\text {int }}=0.030$

\subsection{Refinement}

$R\left[F^{2}>2 \sigma\left(F^{2}\right)\right]=0.042$

$w R\left(F^{2}\right)=0.116$

$S=1.09$

1157 reflections

73 parameters

$\mathrm{H}$-atom parameters constrained

$\Delta \rho_{\max }=0.21{\mathrm{e} \AA^{-3}}^{-3}$

$\Delta \rho_{\min }=-0.24$ e $\AA^{-3}$

Data collection: APEX2 (Bruker, 2009); cell refinement: SAINT (Bruker, 2009); data reduction: $S A I N T$; program(s) used to solve structure: SHELXTL (Sheldrick, 2008); program(s) used to refine structure: SHELXTL; molecular graphics: DIAMOND (Brandenburg, 2010); software used to prepare material for publication: SHELXTL.

\section{Acknowledgements}

This research was supported by the Basic Science Research Program through the National Research Foundation of Korea (NRF) funded by the Ministry of Education, Science and Technology (No. 2014R1A1A4A01009105).

Supporting information for this paper is available from the IUCr electronic archives (Reference: WM5163).

\section{References}

Brandenburg, K. (2010). DIAMOND. Crystal Impact GbR, Bonn, Germany. Bruker (2009). APEX2, SAINT and SADABS. Bruker AXS Inc., Madison, Wisconsin, USA.

Furukawa, H., Kim, J., Ockwig, N. W., O'Keeffe, M. \& Yaghi, O. M. (2008). J. Am. Chem. Soc. 130, 11650-11661.

Paek, K., Knobler, C. B., Maverick, E. F. \& Cram, D. J. (1989). J. Am. Chem. Soc. 111, 8662-8671.

Sheldrick, G. M. (2008). Acta Cryst. A64, 112-122.

Shimizu, S., Nakano, S., Kojima, A. \& Kobayashi, N. (2014). Angew. Chem. Int. Ed. 53, 2408-2412.

Shimizu, S., Zhu, H. \& Kobayashi, N. (2011). Chem. Commun. 47, 3072-3074.

Wu, L.-L., Yang, C.-H., Sun, I.-W., Chu, S.-Y., Kao, P.-C. \& Huang, H.-H. (2007). Organometallics, 26, 2017-2023. 


\section{supporting information}

Acta Cryst. (2015). E71, o430 [doi:10.1107/S2056989015009561]

\section{Crystal structure of [1,1'-biphenyl]-2,2'-dicarbonitrile}

\section{Gihaeng Kang, Tae Ho Kim, Youngeun Jeon and Jineun Kim}

\section{S1. Experimental}

The title compound was prepared by Suzuki coupling reaction of 2-bromobenzonitrile and 2-cyanophenyl boronic acid in acetonitrile (Wu et al., 2007). Slow evaporation of a solution in acetone/ethyl acetate gave single crystals suitable for Xray analysis.

\section{S2. Refinement}

All H-atoms were positioned geometrically and refined using a riding model with $d(\mathrm{C}-\mathrm{H})=0.95 \AA, U_{\text {iso }}(\mathrm{H})=1.2 U_{\text {eq }}(\mathrm{C})$.

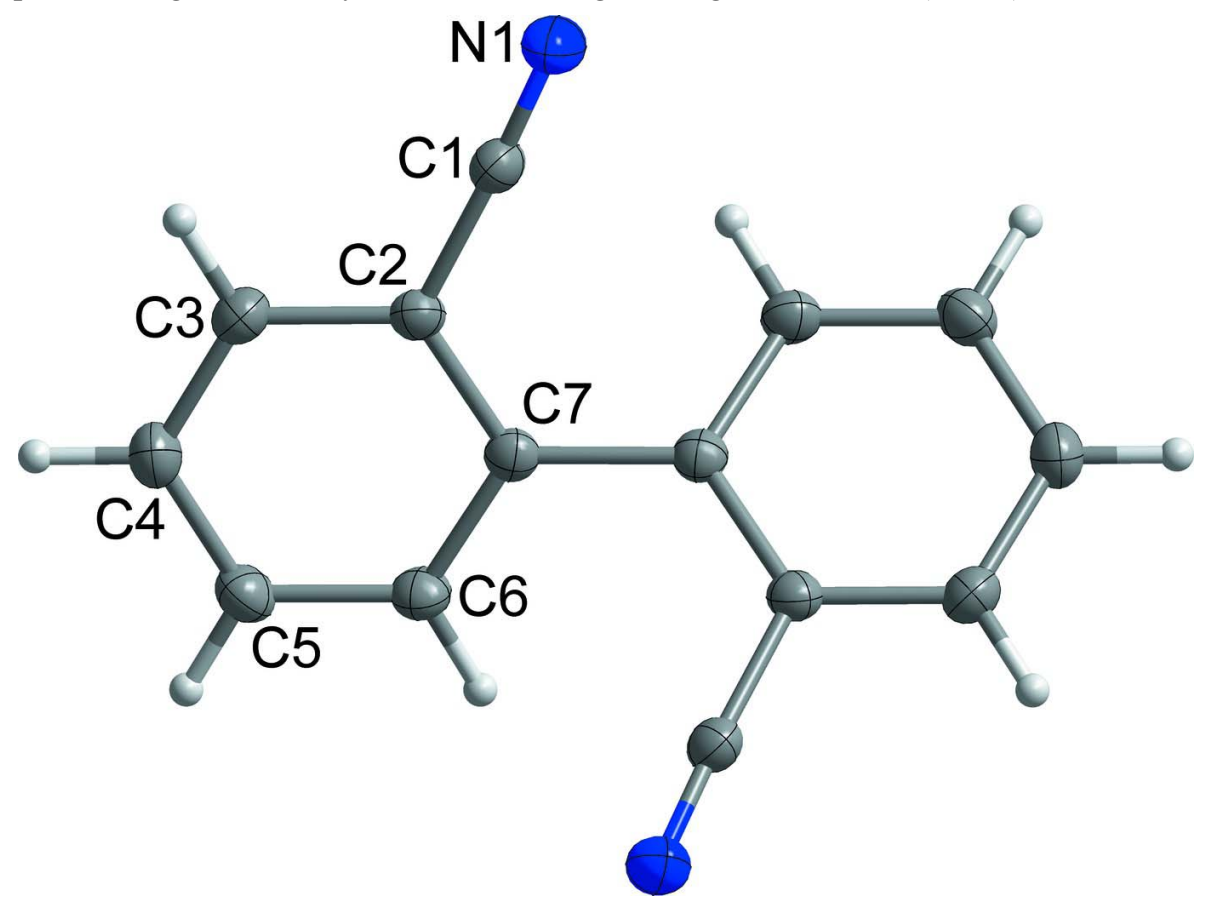

\section{Figure 1}

The molecular structure of the title compound with the atom-numbering scheme. Displacement ellipsoids are drawn at the $50 \%$ probability level. $\mathrm{H}$ atoms are shown as small spheres of arbitrary radius. Symmetry-related atoms (not labelled) are generated by symmetry code $x+1, y,-z+1 / 2$. 


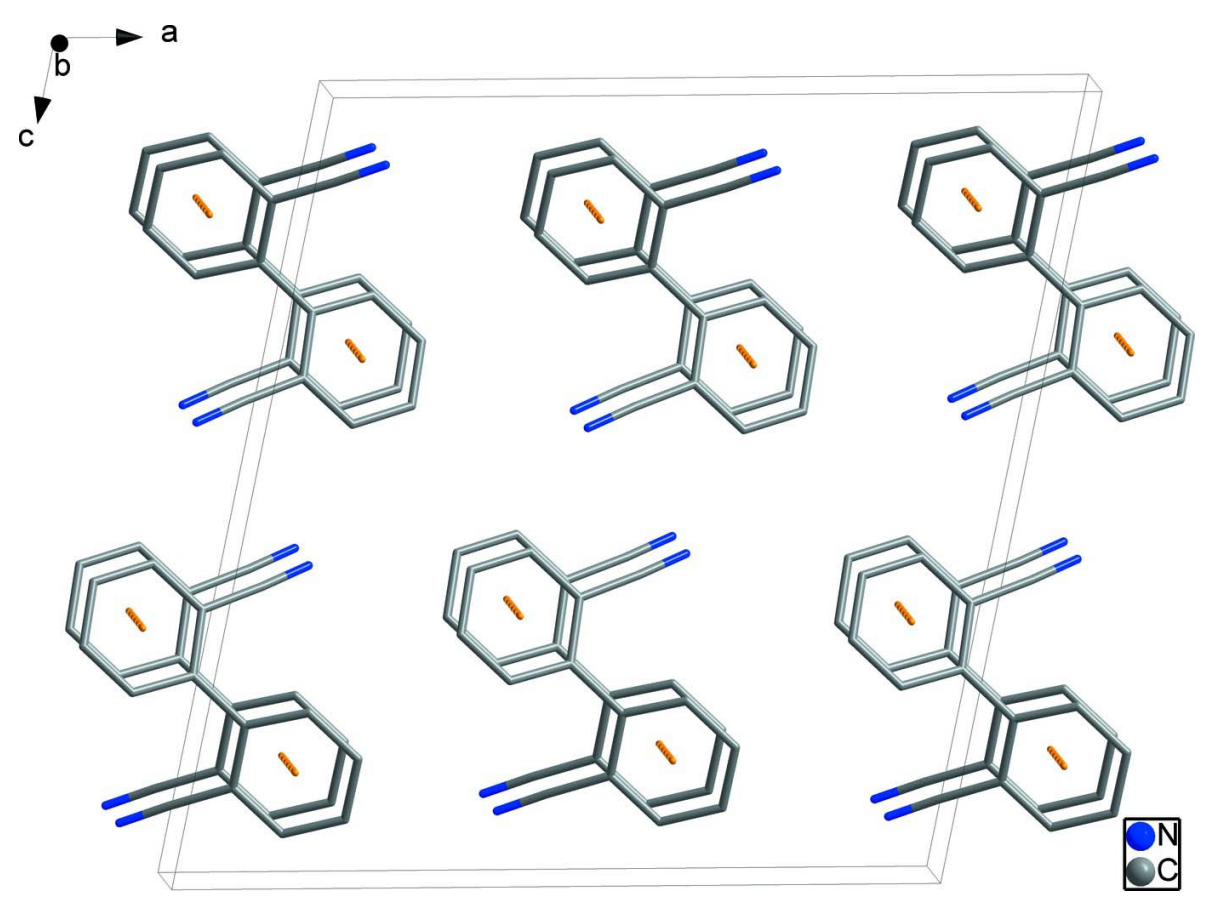

Figure 2

Crystal packing viewed along the $b$ axis. The intermolecular $\pi-\pi$ interactions between the phenyl ring systems $\left[C g 1 \cdots C g 1^{\mathrm{i}}, 3.9451\right.$ (7) $\AA$; $C g 1$ is the centroid of the $\mathrm{C} 2 \cdots \mathrm{C} 7$ ring; symmetry code (i): $\left.x, y-1, z\right]$ are shown as dashed lines. They link molecules into columns along [010].

\section{[1,1'-Biphenyl]-2,2'-dicarbonitrile}

Crystal data

$\mathrm{C}_{14} \mathrm{H}_{8} \mathrm{~N}_{2}$

$M_{r}=204.22$

Monoclinic, $C 2 / c$

$a=15.7839(9) \AA$

$b=3.9451(2) \AA$

$c=16.6079(9) \AA$

$\beta=101.630(3)^{\circ}$

$V=1012.93(9) \AA^{3}$

$Z=4$

Data collection

Bruker APEXII CCD

diffractometer

Radiation source: fine-focus sealed tube

Graphite monochromator

$\varphi$ and $\omega$ scans

Absorption correction: multi-scan

(SADABS; Bruker, 2009)

$T_{\min }=0.966, T_{\max }=0.995$
$F(000)=424$

$D_{\mathrm{x}}=1.339 \mathrm{Mg} \mathrm{m}^{-3}$

Mo $K \alpha$ radiation, $\lambda=0.71073 \AA$

Cell parameters from 1375 reflections

$\theta=3.3-27.5^{\circ}$

$\mu=0.08 \mathrm{~mm}^{-1}$

$T=173 \mathrm{~K}$

Block, colourless

$0.43 \times 0.12 \times 0.06 \mathrm{~mm}$

4708 measured reflections

1157 independent reflections

988 reflections with $I>2 \sigma(I)$

$R_{\text {int }}=0.030$

$\theta_{\max }=27.6^{\circ}, \theta_{\min }=3.3^{\circ}$

$h=-20 \rightarrow 20$

$k=-5 \rightarrow 1$

$l=-21 \rightarrow 19$ 


\section{Refinement}

Refinement on $F^{2}$

Least-squares matrix: full

$R\left[F^{2}>2 \sigma\left(F^{2}\right)\right]=0.042$

$w R\left(F^{2}\right)=0.116$

$S=1.09$

1157 reflections

73 parameters

0 restraints

Primary atom site location: structure-invariant direct methods
Secondary atom site location: difference Fourier map

Hydrogen site location: inferred from neighbouring sites

$\mathrm{H}$-atom parameters constrained

$w=1 /\left[\sigma^{2}\left(F_{\mathrm{o}}^{2}\right)+(0.0584 P)^{2}+0.5212 P\right]$ where $P=\left(F_{\mathrm{o}}{ }^{2}+2 F_{\mathrm{c}}{ }^{2}\right) / 3$

$(\Delta / \sigma)_{\max }<0.001$

$\Delta \rho_{\max }=0.21 \mathrm{e} \AA^{-3}$

$\Delta \rho_{\min }=-0.24$ e $\AA^{-3}$

Special details

Geometry. All e.s.d.'s (except the e.s.d. in the dihedral angle between two l.s. planes) are estimated using the full covariance matrix. The cell e.s.d.'s are taken into account individually in the estimation of e.s.d.'s in distances, angles and torsion angles; correlations between e.s.d.'s in cell parameters are only used when they are defined by crystal symmetry. An approximate (isotropic) treatment of cell e.s.d.'s is used for estimating e.s.d.'s involving 1.s. planes.

Refinement. Refinement of $F^{2}$ against ALL reflections. The weighted $R$-factor $w R$ and goodness of fit $S$ are based on $F^{2}$, conventional $R$-factors $R$ are based on $F$, with $F$ set to zero for negative $F^{2}$. The threshold expression of $F^{2}>\sigma\left(F^{2}\right)$ is used only for calculating $R$-factors(gt) etc. and is not relevant to the choice of reflections for refinement. $R$-factors based on $F^{2}$ are statistically about twice as large as those based on $F$, and $R$ - factors based on ALL data will be even larger.

Fractional atomic coordinates and isotropic or equivalent isotropic displacement parameters $\left(\AA^{2}\right)$

\begin{tabular}{lllll}
\hline & $x$ & $y$ & $z$ & $U_{\text {iso }} * / U_{\text {eq }}$ \\
\hline $\mathrm{N} 1$ & $0.58879(7)$ & $0.3650(3)$ & $0.08819(7)$ & $0.0323(3)$ \\
$\mathrm{C} 1$ & $0.53155(8)$ & $0.2522(3)$ & $0.11172(7)$ & $0.0233(3)$ \\
$\mathrm{C} 2$ & $0.45640(7)$ & $0.1152(3)$ & $0.13725(7)$ & $0.0206(3)$ \\
$\mathrm{C} 3$ & $0.37963(8)$ & $0.1099(3)$ & $0.07828(7)$ & $0.0244(3)$ \\
$\mathrm{H} 3$ & 0.3791 & 0.1894 & 0.0242 & $0.029^{*}$ \\
$\mathrm{C} 4$ & $0.30457(8)$ & $-0.0108(3)$ & $0.09854(8)$ & $0.0273(3)$ \\
$\mathrm{H} 4$ & 0.2522 & -0.0139 & 0.0586 & $0.033^{*}$ \\
$\mathrm{C} 5$ & $0.30609(8)$ & $-0.1274(3)$ & $0.17741(8)$ & $0.0257(3)$ \\
$\mathrm{H} 5$ & 0.2545 & -0.2117 & 0.1914 & $0.031^{*}$ \\
$\mathrm{C} 6$ & $0.38225(8)$ & $-0.1224(3)$ & $0.23640(7)$ & $0.0231(3)$ \\
$\mathrm{H} 6$ & 0.3819 & -0.2037 & 0.2902 & $0.028^{*}$ \\
$\mathrm{C} 7$ & $0.45930(7)$ & $-0.0004(3)$ & $0.21832(7)$ & $0.0196(3)$ \\
\hline
\end{tabular}

Atomic displacement parameters $\left(\AA^{2}\right)$

\begin{tabular}{lllllll}
\hline & $U^{11}$ & $U^{22}$ & $U^{33}$ & $U^{12}$ & $U^{13}$ & $U^{23}$ \\
\hline $\mathrm{N} 1$ & $0.0275(6)$ & $0.0450(7)$ & $0.0253(6)$ & $-0.0066(5)$ & $0.0075(4)$ & $0.0013(5)$ \\
$\mathrm{C} 1$ & $0.0244(6)$ & $0.0277(7)$ & $0.0176(6)$ & $-0.0017(5)$ & $0.0034(4)$ & $-0.0014(5)$ \\
$\mathrm{C} 2$ & $0.0208(6)$ & $0.0225(6)$ & $0.0194(6)$ & $-0.0003(4)$ & $0.0059(4)$ & $-0.0015(4)$ \\
$\mathrm{C} 3$ & $0.0258(6)$ & $0.0290(7)$ & $0.0180(6)$ & $-0.0002(5)$ & $0.0037(5)$ & $0.0003(4)$ \\
$\mathrm{C} 4$ & $0.0214(6)$ & $0.0331(7)$ & $0.0255(7)$ & $-0.0011(5)$ & $0.0000(5)$ & $-0.0016(5)$ \\
$\mathrm{C} 5$ & $0.0206(6)$ & $0.0286(6)$ & $0.0286(7)$ & $-0.0028(5)$ & $0.0069(5)$ & $-0.0006(5)$ \\
C6 & $0.0245(6)$ & $0.0239(6)$ & $0.0216(6)$ & $-0.0017(5)$ & $0.0068(5)$ & $0.0020(4)$ \\
C7 & $0.0205(6)$ & $0.0187(6)$ & $0.0199(6)$ & $0.0012(4)$ & $0.0044(5)$ & $-0.0013(4)$ \\
\hline
\end{tabular}


Geometric parameters $\left(\AA,{ }^{\circ}\right)$

\begin{tabular}{llll}
\hline $\mathrm{N} 1-\mathrm{C} 1$ & $1.1443(16)$ & $\mathrm{C} 4-\mathrm{H} 4$ & 0.9500 \\
$\mathrm{C} 1-\mathrm{C} 2$ & $1.4427(16)$ & $\mathrm{C} 5-\mathrm{C} 6$ & $1.3893(17)$ \\
$\mathrm{C} 2-\mathrm{C} 3$ & $1.3963(16)$ & $\mathrm{C} 5-\mathrm{H} 5$ & 0.9500 \\
$\mathrm{C} 2-\mathrm{C} 7$ & $1.4135(16)$ & $\mathrm{C} 6-\mathrm{C} 7$ & $1.3957(16)$ \\
$\mathrm{C} 3-\mathrm{C} 4$ & $1.3800(17)$ & $\mathrm{C} 6-\mathrm{H} 6$ & 0.9500 \\
$\mathrm{C} 3-\mathrm{H} 3$ & 0.9500 & $\mathrm{C} 7-\mathrm{C} 7^{\mathrm{i}}$ & $1.488(2)$ \\
$\mathrm{C} 4-\mathrm{C} 5$ & $1.3839(18)$ & & \\
& & & $120.68(11)$ \\
$\mathrm{N} 1-\mathrm{C} 1-\mathrm{C} 2$ & $176.92(12)$ & $\mathrm{C} 4-\mathrm{C} 5-\mathrm{C} 6$ & 119.7 \\
$\mathrm{C} 3-\mathrm{C} 2-\mathrm{C} 7$ & $121.34(11)$ & $\mathrm{C} 4-\mathrm{C} 5-\mathrm{H} 5$ & 119.7 \\
$\mathrm{C} 3-\mathrm{C} 2-\mathrm{C} 1$ & $116.62(10)$ & $\mathrm{C} 6-\mathrm{C} 5-\mathrm{H} 5$ & $121.38(11)$ \\
$\mathrm{C} 7-\mathrm{C} 2-\mathrm{C} 1$ & $122.02(10)$ & $\mathrm{C} 5-\mathrm{C} 6-\mathrm{C} 7$ & 119.3 \\
$\mathrm{C} 4-\mathrm{C} 3-\mathrm{C} 2$ & $120.04(11)$ & $\mathrm{C} 5-\mathrm{C} 6-\mathrm{H} 6$ & 119.3 \\
$\mathrm{C} 4-\mathrm{C} 3-\mathrm{H} 3$ & 120.0 & $\mathrm{C} 7-\mathrm{C} 6-\mathrm{H} 6$ & $120.90(12)$ \\
$\mathrm{C} 2-\mathrm{C} 3-\mathrm{H} 3$ & 120.0 & $\mathrm{C} 6-\mathrm{C} 7-\mathrm{C} 2$ & $122.09(12)$ \\
$\mathrm{C} 3-\mathrm{C} 4-\mathrm{C} 5$ & $119.57(11)$ & $\mathrm{C} 6-\mathrm{C} 7-\mathrm{C} 7^{\mathrm{i}}$ & \\
$\mathrm{C} 3-\mathrm{C} 4-\mathrm{H} 4$ & 120.2 & $\mathrm{C} 2-\mathrm{C} 7-\mathrm{C} 7^{\mathrm{i}}$ & $-179.21(9)$ \\
$\mathrm{C} 5-\mathrm{C} 4-\mathrm{H} 4$ & 120.2 & & $0.51(17)$ \\
& & & $178.91(11)$ \\
$\mathrm{C} 7-\mathrm{C} 2-\mathrm{C} 3-\mathrm{C} 4$ & $-0.18(18)$ & $\mathrm{C} 5-\mathrm{C} 6-\mathrm{C} 7-\mathrm{C} 7^{\mathrm{i}}$ & $179.27(9)$ \\
$\mathrm{C} 1-\mathrm{C} 2-\mathrm{C} 3-\mathrm{C} 4$ & $-178.65(11)$ & $\mathrm{C} 3-\mathrm{C} 2-\mathrm{C} 7-\mathrm{C} 6$ & $-2.34(15)$ \\
$\mathrm{C} 2-\mathrm{C} 3-\mathrm{C} 4-\mathrm{C} 5$ & $-0.26(19)$ & $\mathrm{C} 1-\mathrm{C} 2-\mathrm{C} 7-\mathrm{C} 6$ & \\
$\mathrm{C} 3-\mathrm{C} 4-\mathrm{C} 5-\mathrm{C} 6$ & $0.34(19)$ & $\mathrm{C} 3-\mathrm{C} 2-\mathrm{C} 7-\mathrm{C} 7^{\mathrm{i}}$ & \\
$\mathrm{C} 4-\mathrm{C} 5-\mathrm{C} 6-\mathrm{C} 7$ & $0.02(19)$ & $\mathrm{C} 1-\mathrm{C} 2-\mathrm{C} 7-\mathrm{C} 7^{\mathrm{i}}$ & \\
$\mathrm{C} 5-\mathrm{C} 6-\mathrm{C} 7-\mathrm{C} 2$ & $-0.44(17)$ & & \\
& & & \\
\hline $\mathrm{S}$ & & & \\
\hline
\end{tabular}

Symmetry code: (i) $-x+1, y,-z+1 / 2$. 